Castro, CS, Holzgrefe Jr, JV, Reis, RB, Andrade, BB \& Quintanilha, LF (2020). COVID-19 pandemic: scenario of the Brazilian health system for coping with the crisis. Research, Society and Development, 9(7): 1-8, e516974383.

\title{
Pandemia da COVID-19: cenário do sistema de saúde brasileiro para o enfrentamento
} da crise

COVID-19 pandemic: scenario of the Brazilian health system for coping with the crisis Pandemia COVID-19: escenario del sistema de salud brasileño para enfrentar la crisis

Recebido: 10/05/2020 | Revisado: 13/05/2020 | Aceito: 14/05/2020 | Publicado: 24/05/2020

Catarina Sampaio de Castro

ORCID: https://orcid.org/0000-0002-6386-5082

Centro Universitário FTC (UniFTC), Brasil

E-mail: csampaiocastro@gmail.com

José Vaz Holzgrefe Júnior

ORCID: https://orcid.org/0000-0002-6988-767X Escola Bahiana de Medicina e Saúde Pública EBMSP), Brasil E-mail:joseholzgrefe@gmail.com

Renato Barbosa Reis

ORCID: https://orcid.org/0000-0002-2045-112X

Universidade Salvador (UNIFACS), Brasil

E-mail: georeis@gmail.com

Bruno Bezerril Andrade

ORCID: https://orcid.org/0000-0001-6833-3811 Instituto Gonçalo Moniz, Fundação Oswaldo Cruz, Brasil

E-mail: bruno.andrade@fiocruz.br

Luiz Fernando Quintanilha

ORCID: https://orcid.org/0000-0001-8911-9806

Universidade Salvador (UNIFACS), Brasil

E-mail: quintanilha.lf@gmail.com

\section{Resumo}

Objetivo: Este estudo visa descrever e analisar a capacidade operacional dos Estados Brasileiros para o enfrentamento da COVID-19. Metodologia: Foram levantados dados da quantidade de médicos (intensivistas e pneumologistas), leitos de UTI e respiradores em cada 
unidade federativa do país e, em seguida, foi avaliada a relação da oferta desses fatores com as demandas regionais. A taxa de letalidade da doença foi calculada quinze dias após o registro do $1^{\circ}$ óbito por COVID-19, relacionando-a com a capacidade regional de enfrentamento da pandemia. Resultados: Evidenciou-se que há discrepâncias na oferta de médicos, leitos de UTI e ventiladores mecânicos para o atendimento das demandas regionais oriundas da pandemia. O Distrito Federal se destaca como a unidade federativa com a melhor relação de médicos e equipamentos hospitalares para o enfrentamento da crise, enquanto estados do norte e nordeste apresentam, majoritariamente, relações piores que as médias nacionais. Esta distribuição desigual parece interferir na condição de enfrentamento da doença com a possibilidade de impactar nas taxas de letalidade pela COVID-19. Conclusão: Existe uma discrepância da disponibilidade de recursos humanos e equipamentos hospitalares no país sugerindo uma capacidade de enfrentamento desigual da crise que pode impactar nas taxas de letalidade pela COVID-19 e colapso do sistema de saúde.

Palavras-chave: Infecções por coronavírus; Pandemias; Sistema Único de Saúde.

\begin{abstract}
Aims: This study aims to describe and analyze the operational capacity of Brazilian states to face COVID-19. Methodology: Data about the number of physicians (intensivists and pulmonologists), ICU beds and respirators in each federal unit in the country were assessed, and then the relationship between the supply of these factors and regional demands was assessed. The disease lethality rate was calculated fifteen days after the first death was recorded by COVID-19, relating it to the regional capacity to manage with the pandemic. Results: It was evident that there are discrepancies in the supply of doctors, ICU beds and mechanical ventilators in order to attend the regional demands arising from the pandemic. The Federal District stands out as the federative unit with the best ratio of doctors and hospital equipment to face the crisis, while states in the North and Northeast in general have relations worse than the national averages. This unequal distribution seems to interfere in the condition of managing with the disease with the possibility of impacting the mortality rates by COVID19. Conclusion: There is a discrepancy in the availability of human resources and medical equipment in the country suggesting an unequal capacity to face the crisis that can impact the lethality rates by COVID-19 and the collapse of the health system.
\end{abstract}

Keywords: Coronavirus infections; Pandemics; Unified Health System. 


\section{Resumen}

Objetivo: Este estudio tiene como objetivo describir y analizar la capacidad operativa de los estados brasileños para enfrentar COVID-19. Metodología: Se recopilaron datos sobre el número de médicos (intensivistas y pneumólogos), camas de UCI y respiradores en cada unidad federal en el país, y luego se evaluó la relación entre el suministro de estos factores y las demandas regionales. La tasa de letalidad de la enfermedad se calculó quince días después de que COVID-19 registrara la primera muerte, en relación con la capacidad regional para hacer frente a la pandemia. Resultados: se evidenció que existen discrepancias en el suministro de médicos, camas de UCI y ventiladores mecánicos para satisfacer las demandas regionales derivadas de la pandemia. El Distrito Federal se destaca como la unidad federativa con la mejor proporción de médicos y equipos hospitalarios para enfrentar la crisis, mientras que los estados del norte y noreste tienen, in general, relaciones peores que los promedios nacionales. Esta distribución desigual parece interferir en la condición de hacer frente a la enfermedad con la posibilidad de afectar las tasas de mortalidad por COVID-19. Conclusión: Existe una discrepancia en la disponibilidad de recursos humanos y equipos hospitalarios en el país, lo que sugiere una capacidad desigual para hacer frente a la crisis, lo que puede afectar las tasas de mortalidad causadas por COVID-19 y el colapso del sistema de salud.

Palabras Claves: Infecciones por coronavirus; Pandemia; Sistema Unico de Salud.

\section{Introdução}

A atual pandemia da COVID-19 causada pelo novo coronavírus (SARS-CoV-2) tem fomentado uma busca incansável por estratégias de enfrentamento da doença por parte de governantes, cientistas e profissionais da saúde em todo o mundo. A sua descoberta ocorreu no final de dezembro de 2019 em Wuhan, China (WHO, 2020a) e logo se espalhou por vários continentes devido a alguns fatores como a inexperiência para contenção precoce, ausência de tratamento específico e às altas taxas de transmissibilidade, incluindo as transmissões por indivíduos assintomáticos (Bai et al., 2020; Rothe et al., 2020; Shi et al., 2020; Sousa et al., 2020). Desse modo, em pouco mais de quatro meses, o vírus já estava distribuído de forma global, acentuadamente na Europa e América do Norte, com mais de duzentos mil óbitos associados, o que impactou profundamente os sistemas de saúde em países como Itália, Espanha, Reino Unido, Estados Unidos e, mais recentemente, Brasil (WHO, 2020b).

O SARS-CoV-2 tem um período de incubação de 4-5 dias e sua infecção pode se desenvolver de maneira assintomática ou manifestar-se principalmente com febre, tosse seca e 
mialgia (Guan et al., 2020; Shi et al., 2020). Nesse cenário, apesar da maioria dos infectados não apresentarem sintomas ou reportarem sinais da doença de maneira leve, estima-se que aproximadamente 5\% dos casos desenvolvem Síndrome Respiratória Aguda Grave (SARS) com necessidade de serem admitidos em unidade de terapia intensiva (UTI) e de receberem suporte ventilatório. Dos pacientes que evoluem para os casos mais graves, a maioria são idosos, portadores de comorbidades como hipertensão arterial sistêmica e diabetes mellitus. Apesar dos esforços globais, ainda não foram desenvolvidas medidas preventivas mais eficazes como vacinas, e o tratamento é apenas de suporte e inespecífico (Sousa et al., 2020; Zhou et al., 2020).

O Brasil, por meio de seu Ministério da Saúde (MS) ativou, no final de janeiro de 2020, o Centro de Operações de Emergências em Saúde Pública para o SARS-CoV-2, coordenado pela Secretaria de Vigilância em Saúde, com o objetivo de nortear as ações do MS na resposta à possível emergência de saúde pública do Sistema Único de Saúde (SUS). Cerca de um mês depois, no dia 26 de fevereiro, a cidade de São Paulo confirmou o primeiro caso da doença, atingindo posteriormente o espectro de transmissão comunitária em todo o território nacional.

Fez-se necessário, portanto, iniciar a fase de contenção que consiste em evitar a propagação descontrolada do vírus e ganhar tempo para equipar os serviços de saúde com os condicionantes mínimos de atendimentos: leitos hospitalares, suprimento de insumos e equipamentos (EPIs, testes laboratoriais, respiradores). As medidas dessa fase incluem precauções básicas orientadas à população como higiene frequente das mãos, etiqueta respiratória, restrição de viagens além de medidas mais rígidas como o distanciamento social ampliado que inclui trabalho remoto, suspensão em todos os níveis das aulas escolares presenciais, fechamento do comércio físico e serviços não essenciais.

A partir do registro dos 100 primeiros casos confirmados do SARS-CoV-2, instaurouse a fase de mitigação dos riscos que são ações voltadas para evitar agravamento e óbitos. Assim, são estabelecidos fatores mínimos fundamentais para o atendimento dos casos complicados: leitos de UTI com ventilador mecânico e outros tipos de oxigenoterapia suplementar, além de equipe multidisciplinar da saúde incluindo profissionais médicos especializados em terapia intensiva e pneumologia (Anderson et al., 2020; Kucharski et al., 2020).

Este estudo visa descrever e analisar a capacidade operacional dos Estados Brasileiros para o enfrentamento da COVID-19. 
O Brasil possui pouca experiência com crises de saúde pública como é o caso desta que é causada pelo Coronavírus e, diferente dos países europeus e asiáticos, não possui uma cultura local para prevenção dessas situações (Lima et al., 2020). Nesse contexto, o presente estudo tem o objetivo de descrever e analisar a capacidade operacional do Brasil e suas unidades federativas para o enfrentamento da COVID-19. Nele, são avaliados fatores como o número de profissionais médicos especialistas, a quantidade/disponibilidade de leitos de UTI e equipamentos específicos, necessários para o enfrentamento dessa crise de saúde pública.

\section{Metodologia}

Trata-se de um estudo ecológico, descritivo e analítico, a partir de dados secundários como preconiza Pereira et al. (2018). Inicialmente, foram consultados o total de médicos existentes nas especialidades de medicina intensiva e pneumologia de cada Estado Brasileiro. A opção por essas especialidades relacionou-se com o grau de importância no manejo dos casos mais graves da COVID-19. Em seguida, foram quantificados os leitos de UTI (UTI adulto I, II e III) e equipamentos hospitalares (ventiladores mecânicos), essenciais no tratamento das complicações da doença. Esses dados, atualizados em fevereiro de 2020, foram obtidos por meio de consulta na base de dados do CNES (Cadastro Nacional de Estabelecimentos de Saúde), disponibilizados pelo DATASUS (Ministério da Saúde, 2020a).

Com a finalidade de relacionar a oferta de médicos, leitos de UTI e respiradores mecânicos e a população mais exposta à doença fizemos o levantamento, além da população total, do número de indivíduos com idade $\geq 20$ anos no ano de 2020 por meio da tabela de Projeções da População por sexo e idade - Brasil: 2000-2060 e Unidades da Federação 2000-2030, disponibilizada pelo IBGE (IBGE, 2020). Posteriormente, calculamos razão entre a demanda populacional sobre a oferta de médicos e equipamentos hospitalares (x1000). Esses dados foram tabulados, normalizados e serviram de base para a construção de um heatmap em escala de cinza pelo Microsoft Excel - Office 365 a fim de visualizar a distribuição dos recursos humanos e equipamentos hospitalares no país.

Com o objetivo de comparar as condições de enfrentamento entre as Unidades Federativas do país, sua relação com o número de óbitos e a taxa de letalidade pela COVID19, foi feito o levantamento do número de óbitos oficialmente confirmados por SARS-CoV-2 em cada estado (Ministério da Saúde, 2020b). Para essa coleta de dados foi normalizado, em cada unidade federativa, um período de quatorze dias a partir da notificação do primeiro óbito confirmado. Por fim, foi avaliado se existe correlação entre o número de médicos 
especialistas, disponibilidade de leitos de UTI e respiradores com a taxa de letalidade aferida. Para esta análise, utilizamos a correlação de Spearman (two-tailed) com intervalo de confiança de $95 \%$.

Dado que esta pesquisa foi realizada apenas com dados de domínio público, sem a identificação dos envolvidos, não houve a necessidade de apreciação ética.

\section{Resultados}

Existem atualmente no país, cadastrados no Ministério da Saúde com atualização em fevereiro de 2020, 3.139 intensivistas e 1.749 pneumologistas. Este número é mais expressivo na região Sudeste (mais de $50 \%$ do total em ambas as especialidades), especialmente nos Estados Brasileiros de São Paulo e Rio de Janeiro. Por outro lado, este número é menor nos Estados da região Norte. De maneira similar, os equipamentos de saúde mais prioritários no enfrentamento da crise provocada pelo SARS-CoV-2 (leitos de UTI e respiradores) também apresentaram números discrepantes entre os estados. São Paulo, o estado mais populoso do país, conta oficialmente com 18.548 unidades de respiradores mecânicos, seguido do Rio de Janeiro com 7.527. Somente esses dois estados juntos possuem cerca de $40 \%$ do total desses equipamentos no país. No outro extremo, o estado do Amapá conta com apenas 94 (0,14\%). O número de leitos de UTI segue a mesma lógica de distribuição. São Paulo e Rio de Janeiro, novamente, detém cerca de $40 \%$ do total de leitos, enquanto Roraima, com 25 unidades, representa apenas $0,08 \%$ do total (Tabela 1 ). 
Tabela 1: Tabela descritiva do número absoluto de médicos especialistas (intensivistas e pneumologistas) e equipamentos hospitalares (leitos de UTI e respiradores) disponíveis no Brasil.

\begin{tabular}{|c|c|c|c|c|c|c|c|c|c|}
\hline \multirow{2}{*}{ Região } & \multirow{2}{*}{$\mathbf{U F}$} & \multicolumn{2}{|c|}{ Médicos intensivistas } & \multicolumn{2}{|c|}{ Médicos pneumologistas } & \multicolumn{2}{|c|}{ TOTAL de UTIs } & \multicolumn{2}{|c|}{ Respiradores } \\
\hline & & $\mathbf{n}$ & $\%$ & $\mathbf{n}$ & $\%$ & n & $\%$ & $\mathbf{n}$ & $\%$ \\
\hline \multirow{7}{*}{ 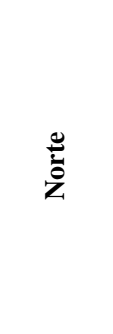 } & $\mathrm{AC}$ & 1 & $0,03 \%$ & 3 & $0,17 \%$ & 48 & $0,16 \%$ & 152 & $0,23 \%$ \\
\hline & $\mathrm{AP}$ & 3 & $0,09 \%$ & 2 & $0,11 \%$ & 46 & $0,15 \%$ & 94 & $0,14 \%$ \\
\hline & $\mathrm{AM}$ & 61 & $1,91 \%$ & 13 & $0,74 \%$ & 271 & $0,88 \%$ & 899 & $1,37 \%$ \\
\hline & PA & 58 & $1,81 \%$ & 34 & $1,94 \%$ & 609 & $1,98 \%$ & 1412 & $2,16 \%$ \\
\hline & RO & 18 & $0,56 \%$ & 8 & $0,46 \%$ & 231 & $0,75 \%$ & 494 & $0,76 \%$ \\
\hline & $\mathrm{RR}$ & 3 & $0,09 \%$ & 4 & $0,23 \%$ & 25 & $0,08 \%$ & 152 & $0,23 \%$ \\
\hline & TO & 3 & $0,09 \%$ & 15 & $0,86 \%$ & 125 & $0,41 \%$ & 318 & $0,49 \%$ \\
\hline \multirow{9}{*}{ 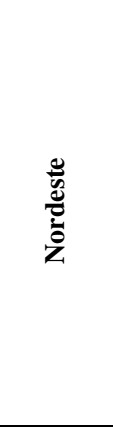 } & $\mathrm{AL}$ & 18 & $0,56 \%$ & 23 & $1,32 \%$ & 299 & $0,97 \%$ & 550 & $0,84 \%$ \\
\hline & BA & 213 & $6,66 \%$ & 105 & $6,00 \%$ & 1478 & $4,80 \%$ & 3194 & $4,88 \%$ \\
\hline & $\mathrm{CE}$ & 91 & $2,84 \%$ & 48 & $2,74 \%$ & 802 & $2,61 \%$ & 2137 & $3,27 \%$ \\
\hline & MA & 33 & $1,03 \%$ & 24 & $1,37 \%$ & 572 & $1,86 \%$ & 1064 & $1,63 \%$ \\
\hline & PB & 79 & $2,47 \%$ & 29 & $1,66 \%$ & 454 & $1,48 \%$ & 918 & $1,40 \%$ \\
\hline & $\mathrm{PE}$ & 184 & $5,75 \%$ & 59 & $3,37 \%$ & 1408 & $4,58 \%$ & 2897 & $4,43 \%$ \\
\hline & PI & 25 & $0,78 \%$ & 11 & $0,63 \%$ & 227 & $0,74 \%$ & 450 & $0,69 \%$ \\
\hline & $\mathrm{RN}$ & 45 & $1,41 \%$ & 20 & $1,14 \%$ & 431 & $1,40 \%$ & 807 & $1,23 \%$ \\
\hline & SE & 11 & $0,34 \%$ & 12 & $0,69 \%$ & 241 & $0,78 \%$ & 511 & $0,78 \%$ \\
\hline \multirow{4}{*}{ 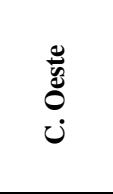 } & DF & 204 & $6,38 \%$ & 65 & $3,72 \%$ & 917 & $2,98 \%$ & 2127 & $3,25 \%$ \\
\hline & GO & 46 & $1,44 \%$ & 48 & $2,74 \%$ & 1053 & $3,42 \%$ & 1728 & $2,64 \%$ \\
\hline & MT & 37 & $1,16 \%$ & 17 & $0,97 \%$ & 592 & $1,92 \%$ & 1404 & $2,15 \%$ \\
\hline & MS & 29 & $0,91 \%$ & 12 & $0,69 \%$ & 352 & $1,14 \%$ & 909 & $1,39 \%$ \\
\hline \multirow{4}{*}{ 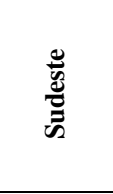 } & ES & 112 & $3,50 \%$ & 50 & $2,86 \%$ & 716 & $2,33 \%$ & 1468 & $2,24 \%$ \\
\hline & MG & 151 & $4,72 \%$ & 150 & $8,58 \%$ & 3096 & $10,06 \%$ & 6263 & $9,57 \%$ \\
\hline & $\mathrm{RJ}$ & 566 & $17,69 \%$ & 259 & $14,81 \%$ & 3978 & $12,93 \%$ & 7527 & $11,51 \%$ \\
\hline & SP & 889 & $27,79 \%$ & 476 & $27,22 \%$ & 8324 & $27,05 \%$ & 18548 & $28,36 \%$ \\
\hline \multirow{3}{*}{$\bar{\Xi}$} & PR & 91 & $2,84 \%$ & 60 & $3,43 \%$ & 2006 & $6,52 \%$ & 3784 & $5,78 \%$ \\
\hline & RS & 128 & $4,00 \%$ & 151 & $8,63 \%$ & 1630 & $5,30 \%$ & 3485 & $5,33 \%$ \\
\hline & $\mathrm{SC}$ & 40 & $1,25 \%$ & 51 & $2,92 \%$ & 843 & $2,74 \%$ & 2119 & $3,24 \%$ \\
\hline TOTAL & BR & 3139 & & 1749 & & 30774 & & 65411 & \\
\hline
\end{tabular}

Fonte: Ministério da Saúde.

Quando se analisam os dados populacionais com a oferta de médicos especialistas, disponibilidade de leitos de UTI e respiradores, o Distrito Federal apresentou os melhores indicadores nos quesitos avaliados, sempre apresentando uma relação de demanda populacional e oferta de médicos e equipamentos hospitalares melhores que as dos outros estados. Por outro lado, estados do Norte e Nordeste se destacam negativamente nesta análise com uma demanda grande para tais itens. Os estados do Acre, Amapá, Piauí e Maranhão, por exemplo, figuram entre os piores indicadores em todas as relações avaliadas (Tabela 2 e Figura 1). Ao estratificarmos a população de 60 anos ou mais, sabidamente mais vulnerável às complicações da COVID-19, o panorama é semelhante (dados não mostrados). 
(CC BY 4.0) | ISSN 2525-3409 | DOI: http://dx.doi.org/10.33448/rsd-v9i7.4383

Tabela 2: Relação entre a população estadual (indivíduos com idade igual ou superior a 20 anos) com o número de médicos especialistas (intensivistas e pneumologistas) e equipamentos hospitalares (leitos de UTI e respiradores) disponíveis no Brasil (x1000). Note a presença de demandas menores no DF em todas as análises realizadas.

\begin{tabular}{|c|c|c|c|c|c|}
\hline Região & $\mathbf{U F}$ & $\begin{array}{c}\text { População ( } \geq 20) \\
\text { / Intensivista }\end{array}$ & $\begin{array}{c}\text { População ( } \geq 20) / \\
\text { Pneumologista }\end{array}$ & $\begin{array}{c}\text { População ( } \geq 20) \\
\text { / UTI adulto }\end{array}$ & $\begin{array}{c}\text { População ( } \geq \mathbf{2 0}) \\
\text { / Respirador }\end{array}$ \\
\hline \multirow{7}{*}{ Zे } & $\mathrm{AC}$ & 544,6 & 181,5 & 11,3 & 3,6 \\
\hline & $\mathrm{AP}$ & 178,5 & 267,8 & 11,6 & 5,7 \\
\hline & $\mathrm{AM}$ & 42,8 & 200,7 & 9,6 & 2,9 \\
\hline & PA & 97,4 & 166,1 & 9,3 & 4,0 \\
\hline & RO & 68,5 & 154,2 & 5,3 & 2,5 \\
\hline & $\mathrm{RR}$ & 136,4 & 102,3 & 16,4 & 2,7 \\
\hline & TO & 356,7 & 71,3 & 8,6 & 3,4 \\
\hline \multirow{9}{*}{ 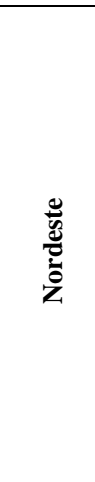 } & $\mathrm{AL}$ & 125,1 & 97,9 & 7,5 & 4,1 \\
\hline & BA & 49,6 & 100,5 & 7,1 & 3,3 \\
\hline & $\mathrm{CE}$ & 71,3 & 135,2 & 8,1 & 3,0 \\
\hline & MA & 140,0 & 192,4 & 8,1 & 4,3 \\
\hline & PB & 36,1 & 98,3 & 6,3 & 3,1 \\
\hline & $\mathrm{PE}$ & 36,5 & 113,9 & 4,8 & 2,3 \\
\hline & PI & 90,5 & 205,7 & 10,0 & 5,0 \\
\hline & $\mathrm{RN}$ & 56,0 & 126,1 & 5,9 & 3,1 \\
\hline & SE & 146,5 & 134,3 & 6,7 & 3,2 \\
\hline \multirow{4}{*}{ 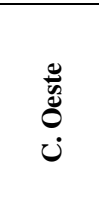 } & DF & 10,8 & 34,0 & 2,4 & 1,0 \\
\hline & GO & 110,1 & 105,5 & 4,8 & 2,9 \\
\hline & MT & 65,9 & 143,4 & 4,1 & 1,7 \\
\hline & MS & 67,7 & 163,6 & 5,6 & 2,2 \\
\hline \multirow{4}{*}{ 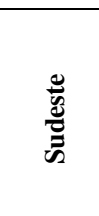 } & ES & 26,1 & 58,6 & 4,1 & 2,0 \\
\hline & MG & 104,7 & 105,4 & 5,1 & 2,5 \\
\hline & RJ & 22,9 & 50,0 & 3,3 & 1,7 \\
\hline & SP & 38,4 & 71,7 & 4,1 & 1,8 \\
\hline \multirow{3}{*}{$\bar{B}$} & PR & 92,3 & 140,0 & 4,2 & 2,2 \\
\hline & RS & 67,0 & 56,8 & 5,3 & 2,5 \\
\hline & $\mathrm{SC}$ & 134,6 & 105,6 & 6,4 & 2,5 \\
\hline \multicolumn{2}{|c|}{ Média Brasil } & 108,0 & 125,3 & 6,9 & 2,9 \\
\hline
\end{tabular}

Fonte: Ministério da Saúde e Instituto Brasileiro de Geografia e Estatística. 
Figura 1: Heatmap com a distribuição geográfica por região e unidades federativas das relações entre o número de Indivíduos adultos com o número de médicos intensivistas, médicos pneumologistas, leitos de UTI e respiradores disponíveis. Os campos pretos representam as maiores demandas e os brancos, as menores. Note as maiores demandas majoritariamente nos estados do Norte e Nordeste (campos mais escuros).

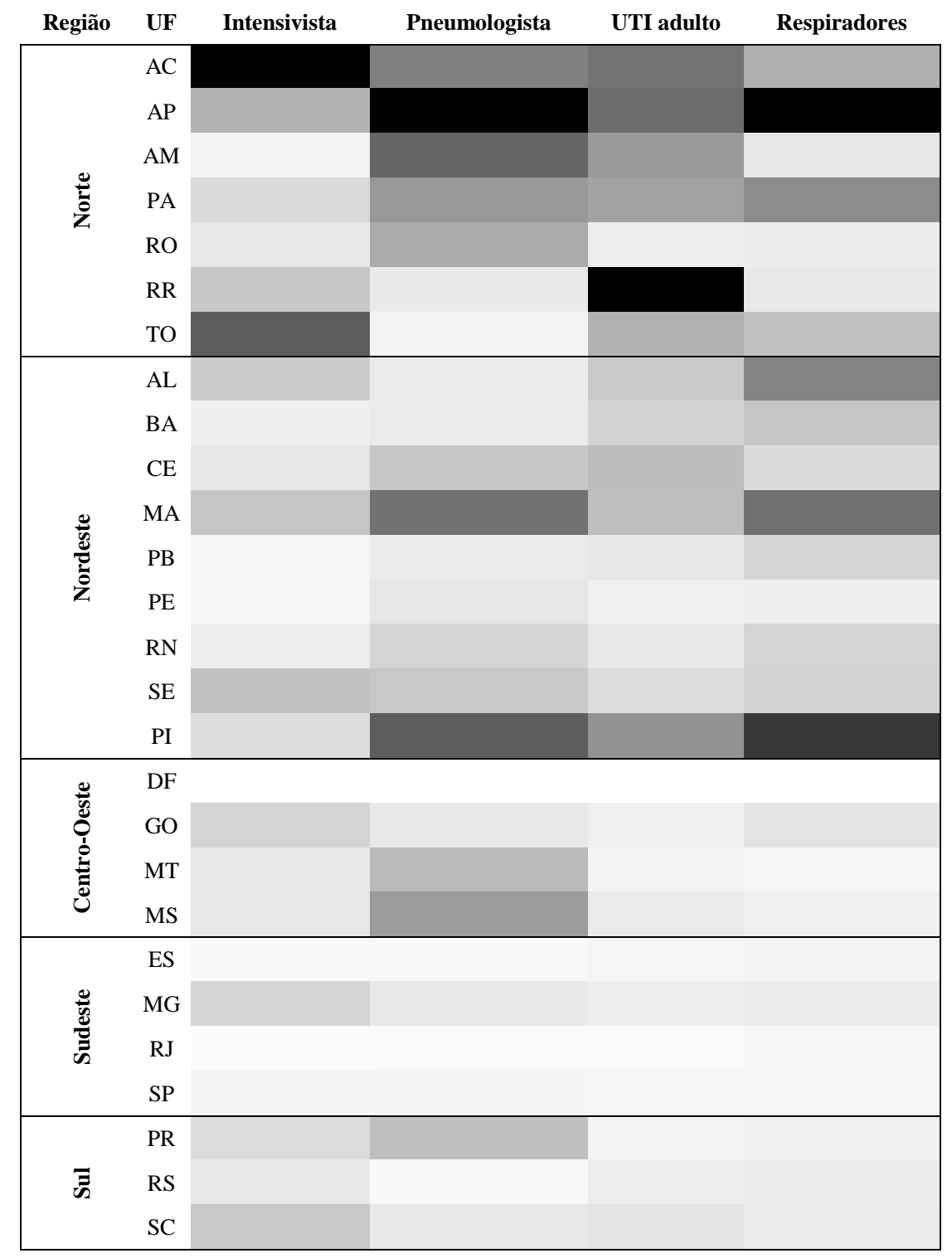

Fonte: Próprios autores.

Considerando as regiões do país, foi evidenciado um descompasso entre a oferta de assistência médica especializada e equipamentos hospitalares para o atendimento geral da população. Nota-se uma maior oferta relativa de assistência médica na região sudeste, enquanto as regiões norte e nordeste apresentam relações inferiores às suas representatividades populacionais em todos os indicadores avaliados (Tabela 3). 
Em relação aos casos, ao considerarmos um período de quatorze dias após a confirmação do primeiro registro por COVID-19 em cada estado, as 27 unidades federativas apresentaram um total de 14.026 casos confirmados de COVID-19. Dentre esses casos, os números foram mais expressivos nos estados de São Paulo $(21,25 \%)$, Ceará $(10,16 \%$,) e Rio de Janeiro $(7,07 \%)$, sendo que as Região Sudeste $(39,51 \%)$ e Nordeste $(25,37 \%)$ foram as que apresentaram maior número de casos, representando quase $2 / 3$ do total. Por outro lado, as regiões Centro-Oeste e Norte foram as que apresentam os números de casos mais reduzidos (Tabela 4). Ainda neste âmbito, o número total de óbitos por COVID-19 em todo o país, durante o mesmo período analisado, foi de 608. O número de óbitos foi maior nos estados de São Paulo (26,97\%), do Ceará $(9,05 \%)$ e Pernambuco (7,57\%), sendo que as Regiões Sudeste $(41,12 \%)$ e Nordeste $(32,89 \%)$ foram, novamente, as que apresentaram maior número de óbitos, representando mais de $70 \%$ dos óbitos no país (Tabela 4). A partir desses dados, a média nacional da taxa de letalidade pela COVID-19 durante o período analisado foi de 4,33\%. Dentre os estados, aqueles com as piores taxas de letalidade foram o Piauí (17,07\%), Paraíba $(13,91 \%)$ e Pernambuco $(11,47 \%)$, sendo que a maioria dos estados da região Nordeste apresentou taxas de letalidade acima da média nacional. Por outro lado, a região Sul $(2,97 \%)$ foi a que apresentou a menor taxa de letalidade (Tabela 4).

Finalmente, avaliamos se a taxa de letalidade se correlaciona com a oferta de médicos e equipamentos hospitalares disponíveis nos estados. Nossos resultados demonstram que, dentre os itens analisados, somente a oferta inadequada de respiradores apresentou uma correlação fracamente positiva com a letalidade $(\mathrm{r}=0.31)$, porém sem representatividade estatística no período avaliado ( $\mathrm{p}=0.11$ ) (Figura 2). 
Tabela3: Relação entre a população geral e o número de equipamentos hospitalares (leitos de UTI e respiradores) e médicos especialistas (intensivistas e pneumologistas) disponíveis nas regiões do Brasil. Note que as distribuições percentuais de recursos humanos e hospitalares são sempre menores nas regiões norte e nordeste quando comparadas com suas representatividades populacionais, enquanto a região sudeste apresenta índices sempre mais robustos que sua demanda populacional.

\begin{tabular}{|c|c|c|c|c|c|c|c|c|c|c|}
\hline Região & Intensivistas & $\%$ & Pneumologistas & $\%$ & UTIs & $\%$ & Respiradores & $\%$ & População & $\%$ \\
\hline Norte & 147 & 4,68 & 79 & 4,52 & 1355 & 4,4 & 3521 & 5,38 & 18.672 .591 & 8,82 \\
\hline Nordeste & 699 & 22,27 & 331 & 18,93 & 5912 & 19,21 & 12528 & 19,15 & 57.374 .243 & 27,09 \\
\hline Centro-Oeste & 316 & 10,07 & 142 & 8,12 & 2914 & 9,47 & 6168 & 9,43 & 16.504 .303 & 7,79 \\
\hline Sul & 259 & 8,25 & 262 & 14,98 & 4479 & 14,55 & 9388 & 14,35 & 30.192 .315 & 14,26 \\
\hline Sudeste & 1718 & 54,73 & 935 & 53,46 & 16114 & 52,36 & 33806 & 51,68 & 89.012 .240 & 42,04 \\
\hline BRASIL & 3139 & 100 & 1749 & 100 & 30774 & 100 & 65411 & 100 & 211.755 .692 & 100 \\
\hline
\end{tabular}

Fonte: Ministério da Saúde e Instituto Brasileiro de Geografia e Estatística. 
Tabela4: Número de casos, óbitos e taxa de letalidade nos estados brasileiros 14 dias após o registro da primeira morte por COVID-19. Note a prevalência de taxas de letalidade maiores que a média nacional nos estados da região Nordeste.

\begin{tabular}{|c|c|c|c|c|c|}
\hline Região & $\mathbf{U F}$ & $\begin{array}{l}\text { Data } 1^{\circ} \text { óbito } \\
\text { registrado }\end{array}$ & $\begin{array}{l}N^{\circ} \text { Casos } \\
\text { após } 14 \text { dias }\end{array}$ & $\begin{array}{l}\mathrm{N}^{0} \text { Óbitos } \\
\text { após } 14 \text { dias }\end{array}$ & $\begin{array}{l}\text { Taxa Letalidade } \\
\text { após } 14 \text { dias }\end{array}$ \\
\hline \multirow{7}{*}{ 苋 } & $\mathrm{AC}$ & $07 / 04 / 2020$ & 195 & 8 & $4,10 \%$ \\
\hline & AP & $31 / 03 / 2020$ & 393 & 10 & $2,54 \%$ \\
\hline & $\mathrm{AM}$ & $04 / 04 / 2020$ & 804 & 30 & $3,73 \%$ \\
\hline & $\mathrm{PA}$ & $25 / 03 / 2020$ & 384 & 21 & $5,47 \%$ \\
\hline & RO & $29 / 03 / 2020$ & 64 & 2 & $3,13 \%$ \\
\hline & $\mathrm{RR}$ & $26 / 03 / 2020$ & 201 & 3 & $1,49 \%$ \\
\hline & TO & $29 / 03 / 2020$ & 116 & 3 & $2,59 \%$ \\
\hline \multirow{9}{*}{ 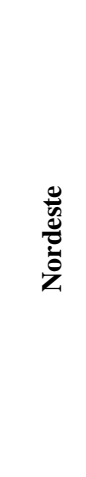 } & $\mathrm{AL}$ & $02 / 04 / 2020$ & 72 & 4 & $5,56 \%$ \\
\hline & BA & $26 / 03 / 2020$ & 673 & 21 & $3,12 \%$ \\
\hline & $\mathrm{CE}$ & $30 / 03 / 2020$ & 1425 & 55 & $3,86 \%$ \\
\hline & MA & 03/04/2020 & 445 & 27 & $6,07 \%$ \\
\hline & $\mathrm{PB}$ & $31 / 03 / 2020$ & 151 & 21 & $13,91 \%$ \\
\hline & $\mathrm{PE}$ & $30 / 03 / 2020$ & 401 & 46 & $11,47 \%$ \\
\hline & PI & $01 / 04 / 2020$ & 41 & 7 & $17,07 \%$ \\
\hline & $\mathrm{RN}$ & $01 / 04 / 2020$ & 302 & 15 & $4,97 \%$ \\
\hline & SE & $27 / 03 / 2020$ & 48 & 4 & $8,33 \%$ \\
\hline \multirow{4}{*}{$\begin{array}{l}\text { 苞 } \\
0 \\
ن\end{array}$} & DF & $25 / 03 / 2020$ & 614 & 14 & $2,28 \%$ \\
\hline & GO & $28 / 03 / 2020$ & 179 & 7 & $3,91 \%$ \\
\hline & MT & $19 / 03 / 2020$ & 162 & 5 & $3,09 \%$ \\
\hline & MS & $29 / 03 / 2020$ & 115 & 4 & $3,48 \%$ \\
\hline \multirow{4}{*}{ 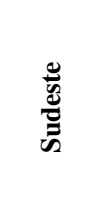 } & ES & $25 / 03 / 2020$ & 754 & 22 & $2,92 \%$ \\
\hline & MG & $31 / 03 / 2020$ & 815 & 23 & $2,82 \%$ \\
\hline & RJ & 04/04/2020 & 992 & 41 & $4,13 \%$ \\
\hline & SP & $26 / 03 / 2020$ & 2981 & 164 & $5,50 \%$ \\
\hline \multirow{3}{*}{$\bar{\Xi}$} & PR & $17 / 03 / 2020$ & 643 & 25 & $3,89 \%$ \\
\hline & RS & $02 / 04 / 2020$ & 555 & 9 & $1,62 \%$ \\
\hline & $\mathrm{SC}$ & $15 / 04 / 2020$ & 501 & 17 & $3,39 \%$ \\
\hline \multicolumn{2}{|c|}{ Brasil } & $17 / 03 / 2020$ & 14026 & 608 & $4,33 \%$ \\
\hline
\end{tabular}

Fonte: Ministério da Saúde. 
Figura 2: Correlação entre as razões de (A) Médicos Intensivistas (B) Pneumologistas, (C) Leitos de UTI e (D) respiradores com a letalidade no período investigado. Note uma correlação fracamente positiva na má distribuição de respiradores e taxa de letalidade no período.

Oferta de Intensivistas $x$ Letalidade COVID-19Oferta de Pneumologistas $x$ Letalidade COVID-19
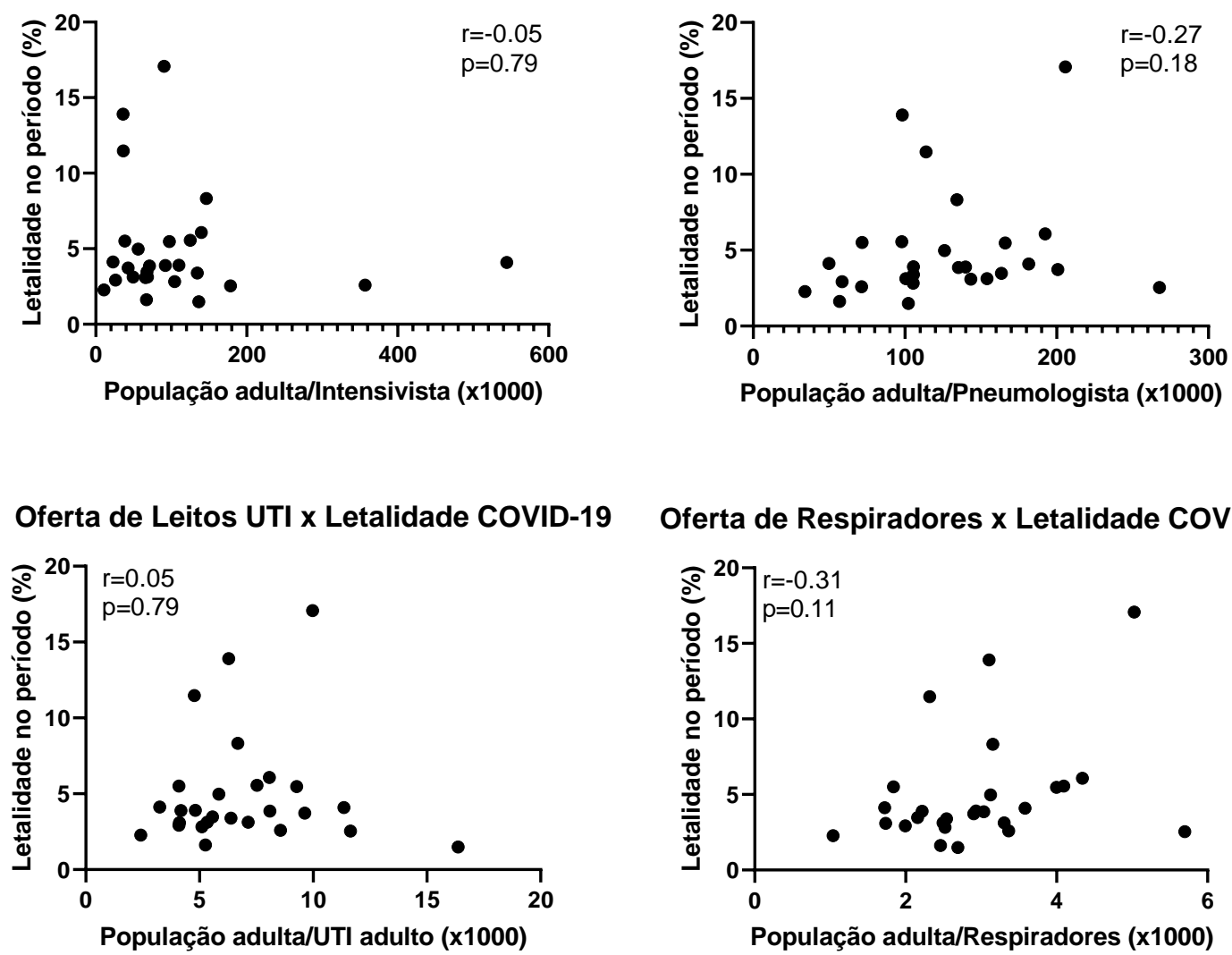

Oferta de Respiradores x Letalidade COVID-19

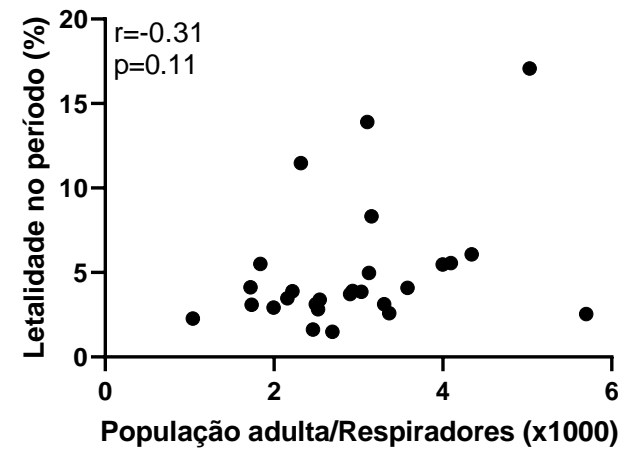

Fonte: Próprios autores.

\section{Discussão}

A atuação no cenário da pandemia da COVID-19 é multidisciplinar incluindo médicos, enfermeiros, fisioterapeutas, além da atuação de diversos outros profissionais. Entretanto, no presente estudo, foram selecionadas as especialidades médicas pneumologia e medicina intensiva por suas relações diretas com características marcantes da COVID-19.

Nos casos que evoluem para SARS o intensivista é o médico mais capacitado para realizar a intubação orotraqueal e manuseio da ventilação mecânica que assegura oxigenação adequada e reduz a taxa de mortalidade por insuficiência respiratória (Anderson et al., 2020; 
Kucharski et al., 2020). O pneumologista atua de forma coadjuvante na avaliação respiratória orientando as melhores estratégias para prevenir dano pulmonar irreversível (Jain, 2020).

O número dos profissionais médicos por unidade federativa foi obtido por meio de dados do Ministério da Saúde. Isto pode ser uma limitação do presente estudo por não sabermos a real abrangência deste número. Entretanto, tais valores foram atualizados em fevereiro de 2020 e, portanto, podem refletir a distribuição desses profissionais no país exatamente no momento do início dos registros de contágio. Outros documentos oficiais como a Demografia Médica do Conselho Federal de Medicina não contêm informações com o nível de apuro e atualização requerida neste estudo.

Os dados aqui apresentados revelam que as condições para o enfrentamento da crise de saúde pública provocado pela COVID-19 são bastante desiguais no país. Enquanto a região Sudeste concentra, proporcionalmente, a maioria absoluta de médicos especialistas e equipamentos hospitalares, as regiões Norte e Nordeste apresentam quantidades inferiores desses parâmetros em relação a sua representatividade populacional.

Para avaliarmos de forma mais fidedigna este cenário, nós optamos por balizar o número de médicos especialistas e equipamentos hospitalares pelo número de indivíduos adultos ( $\geq 20$ anos) de cada estado. Sabe-se que esta população é a mais acometida pela forma grave necessitando de recursos intensivos, sobretudo indivíduos com idade superior a 60 anos que representa a faixa etária mais hospitalizada em 2020 com diagnostico de SARS (Bastos et al., 2020). Foi excluída desse trabalho, portanto, a população infantil que normalmente apresenta quadros muito mais leves da doença com número de óbitos comparativamente desprezíveis (Sociedade Brasileira de Pediatria, 2020).

Na perspectiva da investigação relacionada à população de idosos, o panorama não se altera. A região Sudeste apresenta aporte médico-hospitalar para o atendimento a essa parcela da população proporcionalmente muito superior ao disponível nas regiões Norte e Nordeste do país. Tal cenário fica ainda mais evidente na ocorrência de crises como essa, que exterioriza a realidade já largamente conhecida do Brasil: da intensa desigualdade regional, que inclui o âmbito da saúde.

Em concordância com as necessidades de saúde pública da COVID-19, a oferta de assistência médica no país se mantém irregular e, nessa análise, o Distrito Federal se apresenta como um caso à parte e merece destaque. No centro político do país, todas as relações realizadas se mostraram extremamente favoráveis quando se comparam com os dados de outras unidades federativas, especialmente as das regiões norte e nordeste. 
O SARS-CoV-2 apresenta taxa de letalidade considerável, especialmente em indivíduos idosos portadores de comorbidades, variando de 3.5 a 20\% (Onder et al., 2020). Nesse estudo, a região Sul, com taxa de letalidade de 2,97\%, foi a que apresentou a menor taxa nacional. Por outro lado, apenas estados das regiões Norte e Nordeste e São Paulo apresentaram índices acima da média brasileira no período investigado. Nesse sentido, o estado do Piauí apresentou a pior taxa de letalidade normalizada do país (17,07\%), seguido da Paraíba $(13,91 \%)$ e Pernambuco $(11,47 \%)$, revelando uma possível relação entre a oferta de médicos e equipamentos hospitalares e a taxa de letalidade.

É importante destacar que optamos por calcular a taxa de letalidade em um período comum para todos os estados (14 dias), ou seja, foi contabilizado sempre o número de casos e mortes acumulados duas semanas após o registro do primeiro óbito em cada estado para verificar como o sistema de saúde se comportou no período inicial da crise. Esta normalização foi necessária já que, no momento da elaboração deste estudo, alguns estados já davam sinais de colapso de seus sistemas de saúde, o que poderia comprometer a análise de letalidade.

Neste âmbito, investigamos a correlação entre a oferta de médicos e equipamentos hospitalares para o combate ao COVID-19 com a taxa de letalidade da doença entre os estados. Apesar de a oferta de médicos e leitos de UTI não terem revelado correlação, a inadequada oferta de respiradores se correlacionou fraca, mas positivamente $(r=0.31)$ com a taxa de letalidade sugerindo que o número de respiradores disponíveis, no caso das complicações da COVID-19, pode ser vital para evitar óbitos nos hospitais. Sabe-se, por outro lado, que aspectos como a subnotificação podem afetar diretamente análises como essa permitindo-nos supor que tal correlação pode ser mais intensa do que a apresentada. Além disso, as medidas restritivas tomadas independentemente por cada estado da federação podem, também, contribuir para as diferenças dos números de casos e óbitos aqui apresentados. De qualquer maneira, este trabalho foi capaz de demonstrar que existem diferenças regionais significativas na assistência da saúde da população brasileira o que pode comprometer a capacidade de enfrentamento desta pandemia ou quaisquer outras crises de saúde pública que por ventura se instalarem no país.

O início do desenvolvimento de estratégias efetivas de saúde pública, baseadas em evidência, se dá pela avaliação prévia e generalizada da população (Hallal et al., 2020), portanto é fundamental se atentar para as discrepâncias reveladas neste estudo, visto que os estados que apresentam menores condições para enfrentamento da crise de saúde pública são os mais propensos achegarem rapidamente ao colapso do seu sistema de saúde. 


\section{Considerações Finais}

Existe uma discrepância da disponibilidade de recursos humanos (médicos intensivistas e pneumologistas) e hospitalares (UTIs e ventiladores mecânicos) entre o eixo Sul e Sudeste em relação, sobretudo, ao eixo Norte e Nordeste sugerindo uma capacidade de enfrentamento desigual da crise que pode impactar nas taxas de letalidade pela COVID-19 e colapso do sistema de saúde.

Os dados apresentados neste estudo são de grande relevância para o conhecimento de profissionais de saúde de todo país e podem se tornar uma ferramenta muito útil para gestores públicos de todas as esferas, visando o fortalecimento do SUS através de maiores investimentos e atenção na área da saúde, especialmente nas regiões mais necessitadas, tornando a assistência à saúde uma prática mais homogênea em todo território nacional.

Ademais, esse trabalho, por descrever e analisar o atual cenário brasileiro, revela ser um importante instrumento para tomada de decisões e implementação de novas medidas preventivas de um possível colapso do sistema de saúde frente à COVID-19 nas várias regiões do país.

\section{Referências}

Anderson, RM, Heesterbeek, H, Klinkenberg, D \& Hollingsworth, TD. (2020). How will country-based mitigation measures influence the course of the COVID-19 epidemic? In The Lancet, 21(10228),931-934.

Bai, Y, Yao, L, Wei, T, Tian, F, Jin, DY, Chen, L \& Wang, M. (2020). Presumed Asymptomatic Carrier Transmission of COVID-19. JAMA - Journal of the American Medical Association, 323(14),1406-1407.

Bastos, L. S., Niquini, R. P., Lana, R. M., Villela, D. A. M., Cruz, O. G., Coelho, F. C., Codeço, C. T., \& Gomes, M. F. C. (2020). COVID-19 e hospitalizações por SRAG no Brasil: uma comparação até a 12a semana epidemiológica de 2020. Cadernos de Saúde Pública, 36(4),e00070120.

Guan, W., Ni, Z., Hu, Y., Liang, W., Ou, C., He, J., Liu, L., Shan, H., Lei, C., Hui, D. S. C., Du, B., Li, L., Zeng, G., Yuen, K.-Y., Chen, R., Tang, C., Wang, T., Chen, P., Xiang, J., ... 
Zhong, N. (2020). Clinical Characteristics of Coronavirus Disease 2019 in China. New England Journal of Medicine, 382,1708-1720.

Hallal, P. C., Horta, bernardo L., Barros, A. J. D., Dellagostin, O. A., Hartwig, fernando P., Pellanda, L. C., Struchiner, C. J., Burattini, M. N., Silveira, M. F., Menezes, A. M., Barros, F. C., \& Victora, C. G. (2020). Evolução da prevalência de infecção por COVID-19 no Rio Grande do Sul: inquéritos sorológicos seriados. Revista Ciência \& Saúde Coletiva.

IBGE. (2020). Projeções da população por sexo e idades. Acesso em 02 de abril de 2020, em https://www.ibge.gov.br/estatisticas/sociais/9109-projecao-dapopulacao.html?=\&t=resultados

Jain, A. (2020). COVID-19 and lung pathology. Indian Journal of Pathology and Microbiology, 63(2), 171.

Kucharski, A. J., Russell, T. W., Diamond, C., Liu, Y., Edmunds, J., Funk, S., Eggo, R. M., Sun, F., Jit, M., Munday, J. D., Davies, N., Gimma, A., van Zandvoort, K., Gibbs, H., Hellewell, J., Jarvis, C. I., Clifford, S., Quilty, B. J., Bosse, N. I., ... Flasche, S. (2020). Early dynamics of transmission and control of COVID-19: a mathematical modelling study. The Lancet Infectious Diseases, 20(5),553-558.

Lima, D. L. F., Dias, A. A., Rabelo, R. S., Cruz, I. D. da, Costa, S. C., Nigri, F. M. N., Neri, J. R., Lima, D. L. F., Dias, A. A., Rabelo, R. S., Cruz, I. D. da, Costa, S. C., Nigri, F. M. N., \& Neri, J. R. (2020). COVID-19 no estado do Ceará, Brasil: comportamentos e crenças na chegada da pandemia. Ciência \& Saúde Coletiva, 25(5), 1575-1586.

Ministério de Saúde. (2020a). Informações de Saúde (TABNET). Acesso em 02 de abril de 2020, em http://www2.datasus.gov.br/DATASUS/index.php?area=02

Ministério da Saúde. (2020b). Painel de casos de doença pelo coronavírus 2019 (COVID-19) no Brasil pelo Ministério da Saúde. Acesso em 11 de maio de 2020, em https://covid.saude.gov.br/ 
Onder, G., Rezza, G., \& Brusaferro, S. (2020). Case-Fatality Rate and Characteristics of Patients Dying in Relation to COVID-19 in Italy. JAMA - Journal of the American Medical Association.

Rothe, C., Schunk, M., Sothmann, P., Bretzel, G., Froeschl, G., Wallrauch, C., Zimmer, T., Thiel, V., Janke, C., Guggemos, W., Seilmaier, M., Drosten, C., Vollmar, P., Zwirglmaier, K., Zange, S., Wölfel, R., \& Hoelscher, M. (2020). Transmission of 2019-NCOV infection from an asymptomatic contact in Germany. In New England Journal of Medicine, 382(10),970971.

Shi, Y., Wang, Y., Shao, C., Huang, J., Gan, J., Huang, X., Bucci, E., Piacentini, M., Ippolito, G., \& Melino, G. (2020). COVID-19 infection: the perspectives on immune responses. Cell Death \& Differentiation, 27(5),1451-1454.

Sociedade Brasileira de Pediatria - SBP. (2020). COVID-19 em crianças: envolvimento respiratório. Acesso em 12 de abril, em https://www.sbp.com.br/imprensa/detalhe/nid/covid19-em-criancas-envolvimento-respiratorio/

Sousa, MRN, Barros, SS, Silva, M, Oliveira, APM, Rocha, GM \& Oliveira, GAL. (2020). Patogênese e perspectivas de tratamento da Covid-19: uma revisão. Research, Society and Development, 9(7), 05973730.

WHO - World Health Organization. (2020a). Coronavirus. Acesso em 12 de abril, em https://www.who.int/health-topics/coronavirus\#tab=tab_1

WHO - World Health Organization. (2020b). WHO COVID-19 Dashboard. Acesso em 12 de abril, em https://covid19.who.int/

Zhou, F., Yu, T., Du, R., Fan, G., Liu, Y., Liu, Z., Xiang, J., Wang, Y., Song, B., Gu, X., Guan, L, Wei, Y, Li, H, Wu, X, Xu, J, Tu, S, Zhang, Y, Chen, H \& Cao, B. (2020). Clinical course and risk factors for mortality of adult inpatients with COVID-19 in Wuhan, China: a retrospective cohort study. The Lancet, 395(10229),1054-1062. 
Research, Society and Development, v. 9, n. 7, e516974383, 2020

(CC BY 4.0) | ISSN 2525-3409 | DOI: http://dx.doi.org/10.33448/rsd-v9i7.4383

Porcentagem de contribuição de cada autor no manuscrito

Catarina Sampaio de Castro - $25 \%$

José Vaz Holzgrefe Júnior - 25\%

Renato Barbosa Reis - 12,5\%

Bruno Bezerril Andrade - 12,5\%

Luiz Fernando Quintanilha - 25\% 